\title{
SIMPLE METHOD FOR ESTIMATING MITRAL REGURGITATION BY DYE DILUTION CURVES
}

\author{
BY \\ JOHN SHILLINGFORD* \\ From the Department of Medicine, Postgraduate Medical School of London \\ Received December 2, 1957
}

Stewart (1894), Kinsman et al. (1929), and Hamilton et al. $(1932,1948)$ first showed it possible to estimate cardiac output from a mathematical analysis of dilution curves produced by the passage of an indicator substance through the circulation; good agreement was found between the cardiac output obtained by this and by the direct Fick method. They suggested that the volume between the point of injection and the site of sampling could be calculated from the product of the cardiac output and mean circulation time. More recently we have shown that for a given calculated output and volume the spread of the indicator particles in the curve is fixed within narrow limits (Korner and Shillingford, 1955, 1956). The addition of valvular incompetence in the passage of the indicator substance increases the spread of the particles quite apart from the effect of cardiac output or volume

TIME.

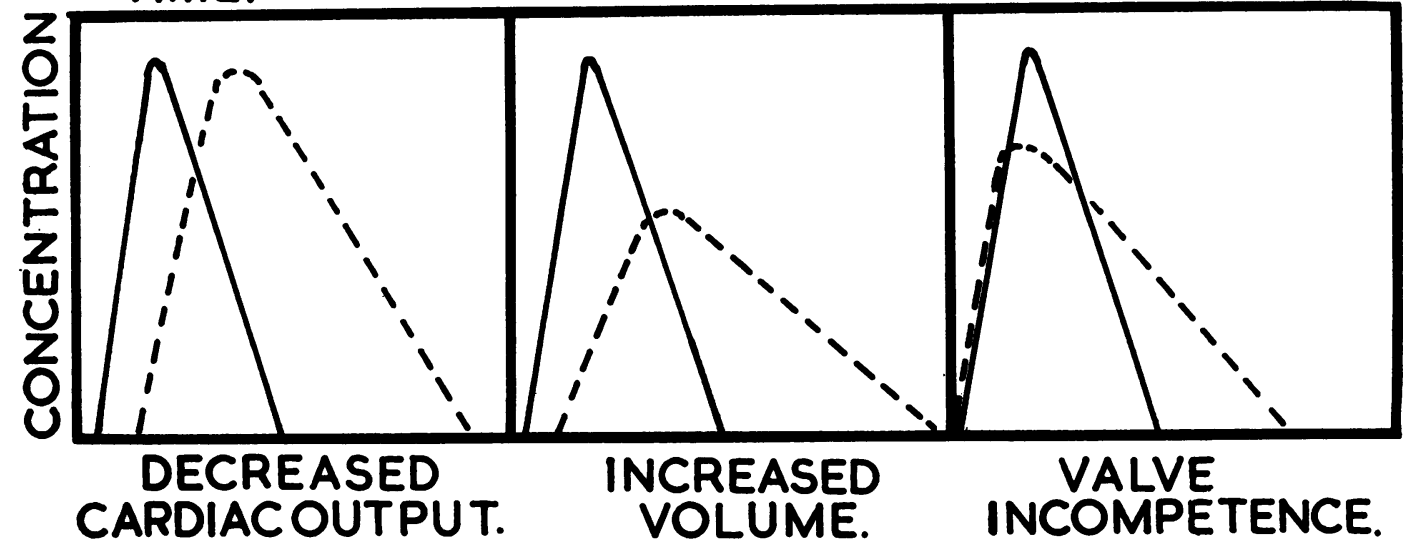

FIG. 1.-Time concentration dilution curves plotted semi-logarithmically to show the effect of cardiac output, volume through which the indicator passes, and valvular incompetence. (The unbroken line represents the reference curve in each case.)

(Fig. 1) and this increase may be used to give an indication of the regurgitant flow through an incompetent valve. We have previously described a method for estimating backflow involving double regression equations (Korner and Shillingford, 1955, 1956). The purpose of this paper is to describe a simple empirical method for obtaining an index of regurgitation from dye curves without involved mathematical calculation.

* Member Scientific Staff, Medical Research Council. 


\section{METHOD}

Evans blue dye (T-1820), 10-20 mg. in 1 to $2 \mathrm{ml}$. normal saline, was rapidly injected into the pulmonary artery or right atrium through a cardiac catheter; pulmonary arterial injection gives the total regurgitant flow through the mitral and aortic valves, and right atrial injection the total through all the cardiac valves. The passage of dye through the ear was recorded by a photo-electric cell earpiece and recorder* based on the design of Wood and Geraci (1949). All patients breathed a high concentration of oxygen from a polythene mask for five minutes before and during the time of recording the dye dilution curves to maintain the blood at constant oxygen saturation.

The spread of the particles in the curve was measured arbitrarily by plotting the curve on semi-logarithmic paper in the normal way used for calculating cardiac output by the Hamilton method and measuring the width of the curve in seconds at a height of one-tenth the maximum concentration (Fig. 2). This figure was divided by the appearance time in seconds to give the ratio of the spread to the appearance time.

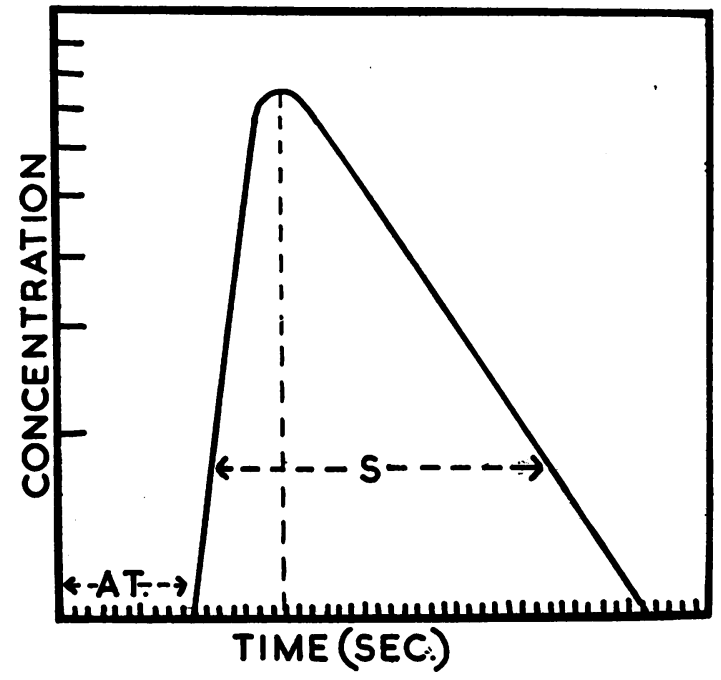

Fig. 2.-The measurements used to determineappearancetime/spread ratio. $\mathrm{AT}=$ appearance time in seconds. $\mathrm{S}=$ spread of curve in seconds at arbitrary figure of one-tenth the maximum concentration (drawn on logarithmic scale).

56 patients, 35 of whom had mitral valve disease with varying amounts of valvular regurgitation, were studied at the time of routine cardiac catheterization. The clinical assessment of the amount of regurgitant flow was based on auscultation of the mitral sounds and systolic murmur, left ventricular size, the pulmonary capillary pressure, and the findings at operation and necropsy. These clinical observations have been found to correspond reasonably well with the regurgitant flow estimated by the variance method previously described and are to be published in full. For the purposes of this paper the patients have been divided into those judged on clinical grounds to have no mitral regurgitation, and those with slight, moderate, and great regurgitation. Where tricuspid and pulmonary regurgitation were clinically absent the dye was injected into the right atrium, but, if they were present, into the pulmonary artery.

\section{RESULTS}

The relationship between the spread/appearance-time ratio in all the patients studied is shown in Fig. 3: it varies between 1.5 and 2.9 in 21 patients without valvular disease. This group includes patients with cardiac outputs varying from 4 to 12 litres a minute and "cardio-pulmonary" volumes from $1 \cdot 1$ to $4 \cdot 1$ litres. In 15 patients with mitral valve disease but judged to have no regurgitation,

* A suitable photo-electric recording instrument can be obtained from Messrs. Cambridge Instruments Company Ltd., Cambridge, England. 


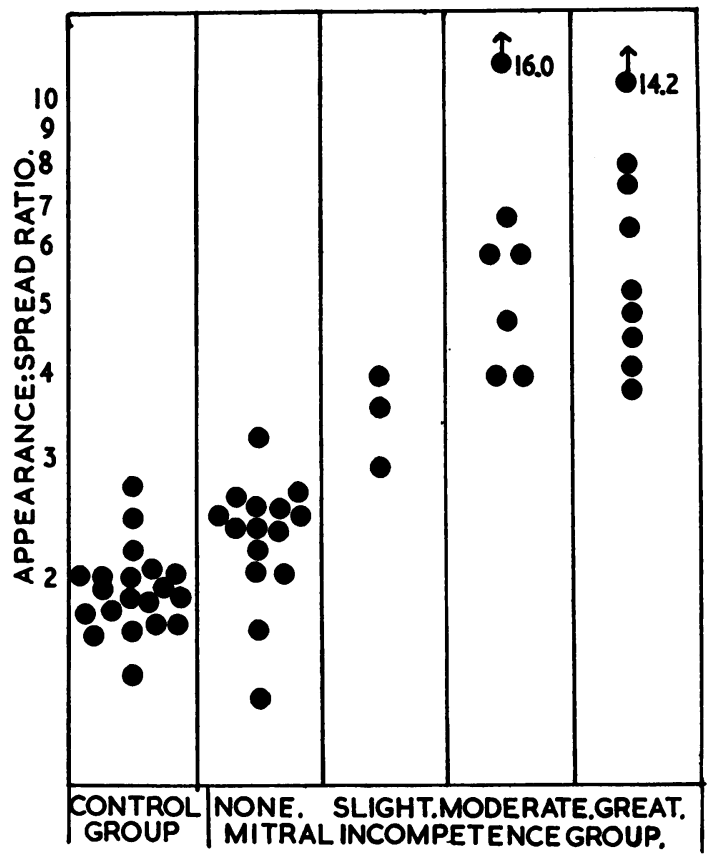

FIG. 3.-The relation between appearancetime/spread of the dye dilution curve and the clinical assessment of mitral regurgitation.

the ratio was within the same limits with the exception of one case where it was $3 \cdot 2$. The figure shows that with increasing valvular regurgitation the ratio becomes greater, and in the severe cases may reach as high as $\mathbf{1 6 \cdot 0}$. In no case where regurgitation was judged to be absent was the ratio over $3 \cdot 2$, and where it was moderate and great the ratio was always over 4.0.

Fig. 4 shows the appearance-time/spread ratio plotted against the regurgitant flow expressed as a percentage of total left ventricular output calculated by the variance method as previously published (Korner and Shillingford, 1956) in the 35 patients with mitral valve disease; as the percentage of regurgitant flow rises the appearance/spread ratio becomes greater.

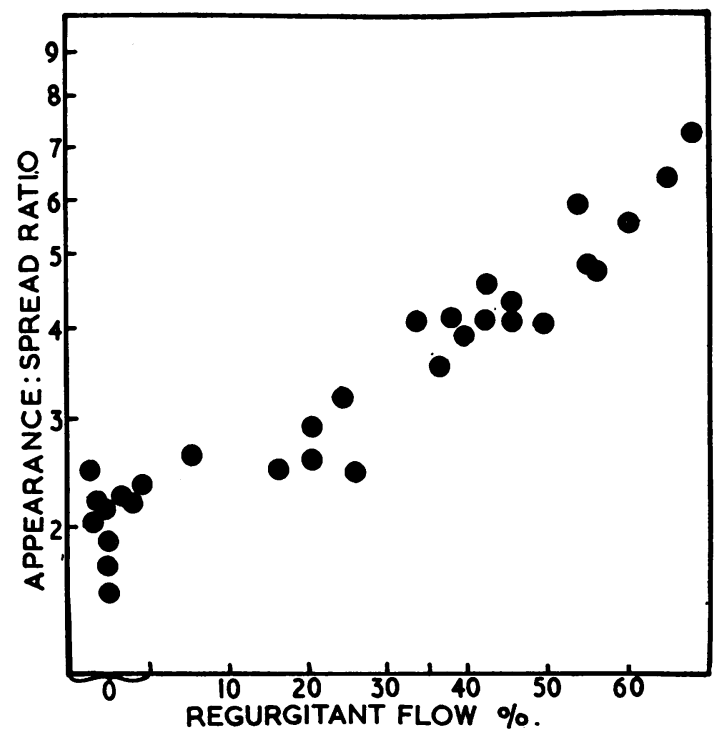

FIG. 4.-Relation between appearance-time/spread ratio and regurgitant flow expressed as a percentage of total left ventricular output calculated by the variance method. 


\section{Discussion}

The validity of this method of estimating backflow depends firstly on the fact that normally the spread of the dye particles increases with the appearance time, so that in the absence of regurgitation at a valve the appearance-time/spread ratio remains relatively constant. Secondly, as we have previously shown (Korner and Shillingford, 1955, 1956), the addition of regurgitation shortens the appearance time and increases the spread of dye particles for any given calculated cardiac output and volume. It seems that this change of appearance-time/spread ratio due to regurgitation is of such a magnitude as to be useful in practice, in gaining additional information on the amount of regurgitation flow in the assessment of patients with mitral regurgitation. If the ratio is 3.0 or under significant regurgitant flow is probably not present, while over 4.0 the regurgitant flow is moderate or greater. With a ratio larger than 6.0 great regurgitation is probably present. Overlap occurs between the groups but any comparison is complicated by the difficulty of clinical and surgical assessment; allowing for this there is a progressive increase of the appearance-time/spread ratio as the estimated backflow increases.

The method has the advantage of great simplicity. Dye can be injected at the time of catheterization and a dilution curve made by the ear recorder. There is no necessity to draw a blood sample and the only calculations needed are to redraw the curve on semi-logarithmic paper and make two measurements that give the appearance-time/spread ratio.

The accuracy and value of this empirical test can only be established by practical experience in a large number of patients. On the basis of this small series it would appear to give reasonable results in mitral valve disease and to enable the absence or presence of significant mitral regurgitation to be established.

It must be emphasized, as we have stated before (Korner and Shillingford, 1955), that it is improbable that dye curves will detect slight amounts of regurgitation. There is also considerable overlap between the groups. In our experience we are finding the method a useful extra test to. supplement and confirm the clinical and hæmodynamic findings in assessing patients with mitral valve disease. Like most tests in clinical medicine it must be used in association with other findings and its limits of error recognized.

\section{SUMMARY}

A simple method for estimating valve regurgitation from dye dilution curves is described.

In a series of patients with mitral valve disease the method has separated those who clinically and surgically had no or slight regurgitation and those with moderate or great regurgitation. The practical value and the limits of error of the test are discussed.

I should like to thank Professor McMichael for his continued help and encouragement, and Dr. Hoffman and Dr.Zoob for their assistance.

\section{REFERENCES}

Hamilton, W. F., Moore, J. W., Kinsman, J. M., and Spurling, R. G. (1932). Amer. J. Physiol., $99,534$.

-, Riley, R. L., Attyah, A. M., Cournand, A., Fowell, D. M., Himmelstein, A., Noble, R. P., Remington, J. W., Richards, D. W., Wheeler, N. C., and Whitham, C. (1948). Amer. J. Physiol., 153, 309.

Kinsman, J. M., Moore, J. W., and Hamilton, W. F. (1929). Amer. J. Physiol., 89, 322.

Korner, P. I., and Shillingford, J. P. (1955). Clin. Sci., 14, 553. , (1956). Clin. Sci., 15, 417.

Stewart, G. N. (1894). J. Physiol., 15, 31.

Wood. E. H., and Geraci, J. E. (1949). J. Lab. clin. Med., 34, 387. 